\title{
Approximations for the Bessel and Airy functions with an explicit error term
}

\author{
Ilia Krasikov
}

\section{ABSTRACT}

We show how one can obtain an asymptotic expression for some special functions with a very explicit error term starting from appropriate upper bounds. We will work out the details for the Bessel function $J_{\nu}(x)$ and the Airy function $\operatorname{Ai}(x)$. In particular, we answer a question raised by Olenko and find a sharp bound on the difference between $J_{\nu}(x)$ and its standard asymptotics. We also give a very simple and surprisingly precise approximation for the zeros $\operatorname{Ai}(x)$.

\section{Introduction and results}

All basic formulas and asymptotic expressions for special functions we use without references can be found in [9]. To write down error terms in a compact form we will use $\theta, \theta_{1}, \theta_{2}, \ldots$, to denote quantities with the absolute value not exceeding one.

In most of the cases error terms of asymptotics of special functions are either not known or, at best, valid for a rather restricted range of parameters. The following is a typical example of that kind (see, for example, [9, Chapter 10]).

The Bessel function $J_{\nu}(x)$ is defined by the series

$$
J_{\nu}(x)=\left(\frac{x}{2}\right)^{\nu} \sum_{j=0}^{\infty}(-1)^{j} \frac{\left(x^{2} / 4\right)^{j}}{j ! \Gamma(j+\nu+1)},
$$

and is a solution of the ODE

$$
x^{2} J_{\nu}^{\prime \prime}(x)+x J_{\nu}^{\prime}(x)+\left(x^{2}-\nu^{2}\right) J_{\nu}(x)=0 .
$$

Theorem 1. Suppose that $\nu \geqslant 0, x>0, \omega_{\nu}=(2 \nu+1) \pi / 4$, and let

$$
\ell_{1} \geqslant \max \left(\frac{\nu}{2}-\frac{1}{4}, 1\right), \quad \ell_{2} \geqslant \max \left(\frac{\nu}{2}-\frac{3}{4}, 1\right), \quad a_{i}(\nu)=\frac{(1 / 2-\nu)_{i}(1 / 2+\nu)_{i}}{2^{i} i !},
$$

then

$$
\begin{aligned}
\sqrt{\frac{\pi x}{2}} J_{\nu}(x)= & \cos \left(x-\omega_{\nu}\right)\left(\sum_{i=0}^{\ell_{1}-1} \frac{a_{2 i}(\nu)}{x^{2 i}}+\theta_{1}^{2} \frac{a_{2 \ell_{1}}(\nu)}{x^{2 \ell_{1}}}\right) \\
& -\sin \left(x-\omega_{\nu}\right)\left(\sum_{i=0}^{\ell_{2}-1} \frac{a_{2 i+1}(\nu)}{x^{2 i+1}}+\theta_{2}^{2} \frac{a_{2 \ell_{2}+1}(\nu)}{x^{2 \ell_{2}+1}}\right) .
\end{aligned}
$$

The assumption $\nu \geqslant 0$ is not really restrictive and can be surmounted by, say, applying the three term recurrence for $J_{\nu}$. However, if $\nu$ is large or depends on $x$, estimating the error term in (3) seems at least as difficult as the original task to find a convenient approximation of the Bessel functions.

In this paper we show how to circumvent this problem and find an explicit expression for error terms, which is uniform in the parameters, provided one has an a priori upper bound

Received 29 July 2013; revised 17 November 2013.

2010 Mathematics Subject Classification 41A60, 33C10 (primary). 
on the absolute value of the considered function. In turn, in many cases, such a bound may be obtained by using so-called Sonin's function. For Bessel and Airy functions, as well as for Hermite polynomials (see $[\mathbf{2}]$ ), the details of this program can be worked out in a quite routine way. For Jacobi and Laguerre polynomials it is a much more involved problem and the result is known only for oscillatory and transition regions [5-7]. It is worth noticing that despite the fact that it is rather a technical problem and we do have appropriate tools to tackle it (see, for example, Lemma 11 and Remark 1 below), one still needs a good deal of calculations to extend the bounds to the monotonicity region. Thus, although the underlying idea of the method we use here is quite simple and can be applied to other special functions satisfying a second order differential equation, it is not utterly straightforward to work out the details. Here we will consider the Bessel function $J_{\nu}(x)$ as an important example to illustrate this approach. We provide asymptotic expressions with an explicit error term for the oscillatory region and also give some new estimates in the monotonicity region. In particular, we answer a question raised by Olenko [8] and find a sharp bound on the difference between $J_{\nu}(x)$ and its standard asymptotics. We also apply the derived results to obtain sharp bounds for the Airy function $\operatorname{Ai}(-x), x>0$. As a corollary we give a surprisingly accurate approximation for its positive zeros.

In what follows it will be convenient to use the following parameters:

$$
\mu=\left|\nu^{2}-\frac{1}{4}\right|, \quad \omega_{\nu}=(2 \nu+1) \pi / 4 .
$$

Let us summarize the main results. First, we will establish a new bound in the monotonicity region which improves the inequality

$$
J_{\nu}(t \nu)<J_{\nu}(\nu) t^{\nu} e^{(1-t) \nu}, \quad \nu>0,0<t<1,
$$

given in $[\mathbf{1 0}]$ and is also stronger than the classical inequality [15, p. 16],

$$
J_{\nu}(x)<\frac{x^{\nu}}{2^{\nu} \Gamma(\nu+1)} e^{-x^{2} / 4(\nu+1)},
$$

provided $x>\sqrt{\ln 16-2} \nu \approx 0.88 \nu$, and $\nu$ is large enough.

Theorem 2. For $\nu>0,0<x \leqslant \nu+\frac{1}{2}$,

$$
J_{\nu}(x)<\frac{2^{1 / 3} x^{\nu}}{3^{2 / 3} \Gamma(2 / 3) \nu^{\nu+1 / 3}} \exp \left(\frac{\nu^{2}-x^{2}}{2 \nu+1}\right) .
$$

The following sharp inequality improves a result obtained in [2] and is crucial for our purposes.

Theorem 3. Let $\nu \geqslant \frac{1}{2}$, then for $x \geqslant 0$,

$$
\left|x^{2}-\mu\right|^{1 / 4}\left|J_{\nu}(x)\right|<\sqrt{2 / \pi},
$$

and the constant $\sqrt{2 / \pi}$ is best possible.

The following theorem provides a bound on the difference between $J_{\nu}(x)$ and its standard asymptotics.

TheOREM 4. For $x>0$,

$$
J_{\nu}(x)=\sqrt{\frac{2}{\pi x}} \cos \left(x-\omega_{\nu}\right)+\theta c \mu x^{-3 / 2},
$$


where

$$
c= \begin{cases}(2 / \pi)^{3 / 2}, & x \geqslant 0,|\nu| \leqslant 1 / 2 \\ 4 / 5, & 0<x<\sqrt{\mu}, \nu>1 / 2 \\ 2 / \pi, & x \geqslant \sqrt{\mu}, \nu>1 / 2\end{cases}
$$

Moreover, up to the numerical factor $c$, the error term is sharp. In particular, $c$ cannot be taken less than $1 / \sqrt{2 \pi}$.

In [8] Olenko proved the inequalities which for $\nu>0$ can be written as

$$
c_{1} \nu^{7 / 6} \leqslant \sup _{x \geqslant 0} x^{3 / 2}\left|J_{\nu}(x)-\sqrt{\frac{2}{\pi x}} \cos \left(x-\omega_{\nu}\right)\right| \leqslant c_{2} \nu^{13 / 6},
$$

with some explicit constants $c_{1}, c_{2}$, and raised the question of what is the best possible exponent $\alpha$ of $\nu$ in these inequalities. The answer $\alpha=2$ is an immediate corollary of (6) and for $\nu \geqslant-\frac{1}{2}$ we obtain

$$
\frac{1}{\sqrt{2 \pi}} \mu \leqslant \sup _{x \geqslant 0} x^{3 / 2}\left|J_{\nu}(x)-\sqrt{\frac{2}{\pi x}} \cos \left(x-\omega_{\nu}\right)\right|<\frac{4}{5} \mu .
$$

The next theorem gives a more complicated yet much sharper approximation for the Bessel function $J_{\nu}(x)$.

THEOREM 5. For $|\nu| \leqslant \frac{1}{2}$ and $x>0$,

$$
J_{\nu}(x)=\sqrt{\frac{2}{\pi}}\left(x^{2}+\mu\right)^{-1 / 4} \cos \left(\mathcal{B}(x)-\omega_{\nu}\right)+\theta \frac{\mu}{\sqrt{2 \pi x}\left(x^{2}+\mu\right)^{3 / 2}},
$$

and for $|\nu|>\frac{1}{2}$ and $x>\sqrt{\mu}$

$$
J_{\nu}(x)=\sqrt{\frac{2}{\pi}}\left(x^{2}-\mu\right)^{-1 / 4} \cos \left(\mathcal{B}(x)-\omega_{\nu}\right)+\theta \frac{13 \mu}{12 \sqrt{2 \pi}\left(x^{2}-\mu\right)^{7 / 4}},
$$

where

$$
\mathcal{B}(x)= \begin{cases}\sqrt{x^{2}+\mu}+\sqrt{\mu} \ln \frac{x}{\sqrt{\mu}+\sqrt{x^{2}+\mu}}, & |\nu| \leqslant 1 / 2, \\ \sqrt{x^{2}-\mu}+\sqrt{\mu} \arcsin \frac{\sqrt{\mu}}{x}, & \nu \geqslant 1 / 2 .\end{cases}
$$

Notice that (9) remains reasonably accurate even in the transition region when $x=\nu+$ const $\cdot \nu^{1 / 3}$.

Formula (9) can be rewritten in a slightly simpler way by setting $x=\sqrt{\mu} / \sin t$,

$$
J_{\nu}(\sqrt{\mu} / \sin t)=\sqrt{\frac{2}{\pi}} \mu^{-1 / 4} \tan t \cos \left((t+\cot t) \sqrt{\mu}-\omega_{\nu}\right)+\theta \frac{13 \tan ^{7 / 2} t}{12 \sqrt{2 \pi} \mu^{3 / 4}} .
$$

The argument of the cosine in (8) can be simplified at the cost of a weaker numerical constant at the error term. 
Theorem 6. For $|\nu| \leqslant \frac{1}{2}$ and $x>0$,

$$
J_{\nu}(x)=\sqrt{\frac{2}{\pi}} \frac{\cos \left(x-\mu / 2 x-\omega_{\nu}\right)}{\left(x^{2}+\mu\right)^{1 / 4}}+\theta \frac{25 \mu}{24 \sqrt{2 \pi} x^{3}\left(x^{2}+\mu\right)^{1 / 4}} .
$$

Let $j_{\nu s}$ be the $s$ th zero of $J_{\nu}(x)$. One can readily derive sharp approximations for $j_{\nu s}$ using formulas (8), (9) and (12), and imposing some restrictions on the rate of growth of $\nu$ and $s$ to be able to solve arising transcendental equations. To illustrate this approach, we derive an error term in the asymptotic McMahon's expansion (see [15, p. 506])

$$
j_{\nu s}=r / 4+2 \mu / r+O\left(s^{-3}\right), \quad r=(4 s+2 \nu-1) \pi \rightarrow \infty .
$$

Corollary 1. For $|\nu| \leqslant \frac{1}{2}$

$$
j_{\nu s}=r / 4+2 \mu / r+18 \pi \theta \mu r^{-3} .
$$

Let us notice that in fact for $|\nu| \leqslant \frac{1}{2}$ a stronger result, yet with a much more involved proof, is known $[\mathbf{3}]$ :

$$
j_{\nu s}=r / 4+2 \mu / r-\frac{8 \mu(7 \mu+6)}{3 r^{3}}+81 \theta^{2} r^{-5} .
$$

Bounds and asymptotics for the Bessel function lead directly to approximation of the Airy function

$$
\operatorname{Ai}(-x)=\frac{\sqrt{x}}{3}\left(J_{-1 / 3}(\zeta)+J_{1 / 3}(\zeta)\right), \quad \zeta=\frac{2 x^{3 / 2}}{3}
$$

In this paper we prove the following.

Theorem 7. For $x>0$,

$$
x^{1 / 4}|\operatorname{Ai}(-x)|<1 / \sqrt{\pi} .
$$

Theorem 8. For $x>0$,

$$
\operatorname{Ai}(-x)=\frac{\cos (\zeta-\pi / 4)}{\sqrt{\pi} x^{1 / 4}}+\theta \frac{5}{24 \pi^{3 / 2} x^{7 / 4}} .
$$

Applying (8) to the Airy function readily yields a much more accurate result.

Corollary 2. For $x>0$,

$$
\begin{aligned}
\operatorname{Ai}(-x)= & \frac{2 \sqrt{x} \cos \left(\sqrt{16 x^{3}+5} / 6-(\sqrt{5} / 6) \ln \left(\left(\sqrt{16 x^{3}+5}+\sqrt{5}\right) / 4 x^{3 / 2}\right)-\pi / 4\right)}{\sqrt{\pi}\left(16 x^{3}+5\right)^{1 / 4}} \\
& +\theta \frac{10 \sqrt{3}}{\sqrt{\pi} x^{1 / 4}\left(16 x^{3}+5\right)^{3 / 2}} .
\end{aligned}
$$

With a slightly less precise numerical constant at the error term this expression can be written in a much simpler form.

Theorem 9. For $x>0$,

$$
\operatorname{Ai}(-x)=\frac{2 \sqrt{x} \cos \left((2 / 3) x^{3 / 2}-(5 / 48) x^{-3 / 2}-\pi / 4\right)}{\sqrt{\pi}\left(16 x^{3}+5\right)^{1 / 4}}+\theta \frac{5}{9 \sqrt{\pi} x^{4}\left(16 x^{3}+5\right)^{1 / 4}} .
$$


Let $a_{s}$ be the $s$ th positive zero of $\operatorname{Ai}(-x)$. In [1] Breen established the bound $a_{s}=$ $\frac{1}{4} \pi^{2 / 3}(12 s-3 \alpha)^{2 / 3}$, where $\alpha \in(2.895,4.2)$. Here we will strengthen this bound and show that formula (17) yields a sharper and also very simple approximation to $a_{s}$, for example already for the first zero the error is less than $\frac{4}{3} \cdot 10^{-3}$.

TheOREM 10. We have

$$
a_{s}=\frac{1}{4}\left(m^{2}+20\right)^{1 / 3}+\theta \frac{457}{m^{3}\left(m^{2}+40\right)^{1 / 6}}, \quad m=(12 s-3) \pi .
$$

The paper is organized as follows. In the next section we describe the idea of the method. In $\S 3$ we establish upper bounds on Bessel and Airy functions, in particular, we prove Theorems 2, 3 and 7. In $\S 4$ we consider the error term of the standard asymptotics and prove Theorem 4, thus answering Olenko's question. The rest of the results will be obtained in $\S 5$, where using the approach of $\S 2$, we establish sharper approximations for Bessel and Airy functions and in $\S 6$, where we prove Corollary 1 and Theorem 10.

\section{Preliminaries}

Our main tool for bounding functions satisfying a second order ODE is based on so-called Sonin's function, which is defined as follows. Let $y(x)$ be a solution of

$$
y^{\prime \prime}(x)+a(x) y^{\prime}(x)+b(x) y(x)=0, b(x)>0 .
$$

Then $S(x)=y^{2}+y^{\prime 2} / b$ is just an envelope of $y^{2}$, coinciding with it in all maxima. The sign of $S^{\prime}=\left(2 a b+b^{\prime}\right) y^{\prime 2} / b^{2}$ depends only on $a$ and $b$, which in many cases enables one to find the global maximum of $|y|$. The following approach was briefly described in [7]. We want to find an approximation of a solution of the differential equation

$$
f^{\prime \prime}+b^{2}(x) f(x)=0,
$$

in terms of some standard function $F(x)$, which also satisfies a second order ODE

$$
\mathcal{D}_{1}(F)=p_{2}(x) F^{\prime \prime}+p_{1}(x) F^{\prime}+p_{0}(x) F=0 .
$$

We seek for a multiplier function $z(x)$ such that the differential operator

$$
\mathcal{D}_{2}(g)=q_{2}(x) g^{\prime \prime}+q_{1}(x) g^{\prime}+q_{0}(x) g,
$$

for $g=g(x)=z(x) f(x)$, is in some sense close to the operator $\mathcal{D}_{1}$. In fact, in what follows we choose $F$ to be just $\cos \phi(x)$ with an appropriate function $\phi$.

To be more specific, consider a WKB-type approximation where one chooses $g(x)=$ $\sqrt{b(x)} f(x)$, transforming (19) into

$$
g^{\prime \prime}-\frac{b^{\prime}}{b} g^{\prime}+b^{2} g(1+\epsilon(x))=0, \quad \epsilon(x)=\frac{3 b^{2}-2 b b^{\prime \prime}}{4 b^{4}} .
$$

If $\epsilon$ is small we can expect that $g(x)$ is close to the solution of the equation

$$
g_{0}^{\prime \prime}-\frac{b^{\prime}}{b} g_{0}^{\prime}+b^{2} g_{0}=0
$$

which is just $g_{0}=M \cos \mathcal{B}(x)$, where $\mathcal{B}(x)=\int b(x) d x$. 
Assume now that we have an a priori bound, say, $|g(x)| \leqslant C$. Then we can readily estimate the error term $\left|g-g_{0}\right|$ by solving (20) as an inhomogeneous equation,

$$
g(x)=g_{0}-\int^{x} \epsilon(t) b(t) \sin (\mathcal{B}(x)-\mathcal{B}(t)) g(t) d t,
$$

thus obtaining

$$
g=g_{0}+\theta C \int^{x} \epsilon(t) b(t) d t
$$

To derive an upper bound on $|g(x)|$ we consider Sonin's function

$$
S(x)=S(g ; x)=g^{2}+\frac{g^{\prime 2}}{(1+\epsilon) b^{2}}=g^{2}+\frac{4 b^{2}}{4 b^{4}+3 b^{\prime 2}-2 b b^{\prime \prime}} g^{\prime 2} .
$$

Applying (20) to get rid of $g^{\prime \prime}$, one finds

$$
S^{\prime}(x)=\frac{8 b\left(6 b^{\prime 3}-6 b b^{\prime} b^{\prime \prime}+b^{2} b^{\prime \prime \prime}\right)}{\left(4 b^{4}+3 b^{\prime 2}-2 b b^{\prime \prime}\right)^{2}} g^{\prime 2} .
$$

Let us assume now that $4 b^{4}+3 b^{2}-2 b b^{\prime \prime}>0$ and $6 b^{\prime 3}-6 b b^{\prime} b^{\prime \prime}+b^{2} b^{\prime \prime \prime}>0$, then $S^{\prime}>0$ and we obtain $g^{2}(x)<S(\infty)$. Moreover, one can also get an upper bound on $S$ in the following way:

$$
S-\frac{b\left(4 b^{4}+3 b^{\prime 2}-2 b b^{\prime \prime}\right)}{2\left(6 b^{\prime 3}-6 b b^{\prime} b^{\prime \prime}+b^{\prime 2} b^{\prime \prime \prime}\right)} S^{\prime}=g^{2} \geqslant 0,
$$

that is

$$
S^{\prime} / S \leqslant \frac{2\left(6 b^{\prime 3}-6 b b^{\prime} b^{\prime \prime}+b^{\prime 2} b^{\prime \prime \prime}\right)}{b\left(4 b^{4}+3 b^{\prime 2}-2 b b^{\prime \prime}\right)}=\frac{d}{d x} \ln \frac{b^{4}}{4 b^{4}+3 b^{\prime 2}-2 b b^{\prime \prime}},
$$

provided the last expression is nonnegative. Integrating from $x$ to $y$, we find

$$
\frac{S(y)}{S(x)} \leqslant \frac{1+\epsilon(x)}{1+\epsilon(y)}, \quad x<y .
$$

Thus, the envelope of $g^{2}(x)$ given by $S(x)$ is almost constant as far as $\epsilon(x)=o(1)$.

In practically important examples the situation is somewhat more subtle as the coefficient $b(x)$ may vanish. For instance, for the Bessel function the coefficient $b(x)=x^{-1} \sqrt{x^{2}-\nu^{2}+\frac{1}{4}}$, and Sonin's function does not provide any information for the monotonicity region $0 \leqslant x \leqslant$ $\sqrt{\nu^{2}-\frac{1}{4}}$. Thus, one needs some supplementary estimates to extend the bounds on $|g(x)|$ to this interval. Let us notice that although the behaviour of the solutions of (19) looks less complicated in the monotonicity region, it probably allows only a piecewise approximation in reasonably simple elementary functions.

Another rather technical problem is how to find the constants of integration in $g_{0}$. Here one either has to know the value of $g(x)$ at some points, for example at infinity, or to be able to match asymptotics in the oscillatory and transition regions.

\section{Upper bounds}

First we will establish a new upper bound on $J_{\nu}(x)$ in the monotonicity region. The simplest inequality of this type [15] states that for $x$ real and $\nu \geqslant-\frac{1}{2}$,

$$
\left|J_{\nu}(x)\right| \leqslant \frac{|x|^{\nu}}{2^{\nu} \Gamma(\nu+1)} .
$$

For our purposes we need much more accurate estimates. We will use the following inequality established in [4]. We sketch a proof for self-completeness. 
Lemma 11. Let $\mathcal{J}_{\nu}(x)=x^{-\nu} J_{\nu}(x), \nu \geqslant-\frac{1}{2}$, then for $0<x \leqslant \nu+\frac{1}{2}$,

$$
\frac{\mathcal{J}_{\nu}^{\prime}(x)}{\mathcal{J}_{\nu}(x)} \geqslant \frac{\sqrt{(2 \nu+1)^{2}-4 x^{2}}-2 \nu-1}{2 x} \geqslant-\frac{2 x}{2 \nu+1} .
$$

Proof. For $\nu \geqslant-\frac{1}{2}$ the Bessel function $\mathcal{J}_{\nu}(x)$ is an entire function with only real zeros satisfying the Laguerre inequality $\mathcal{J}_{\nu}^{\prime 2}-\mathcal{J}_{\nu} \mathcal{J}_{\nu}^{\prime \prime} \geqslant 0$. Substituting $\mathcal{J}_{\nu}^{\prime \prime}$ from the differential equation

$$
x \mathcal{J}_{\nu}^{\prime \prime}+(2 \nu+1) \mathcal{J}_{\nu}^{\prime}+x \mathcal{J}_{\nu}=0
$$

and dividing by $\mathcal{J}_{\nu}^{2}$ we obtain $x t^{2}(x)+(2 \nu+1) t(x)+x \geqslant 0$, where $t(x)=\mathcal{J}_{\nu}^{\prime} / \mathcal{J}_{\nu}$. Hence for $0<x \leqslant \nu+\frac{1}{2}$

$$
t(x) \notin\left(-\frac{\sqrt{(2 \nu+1)^{2}-4 x^{2}}+2 \nu+1}{2 x},-\frac{2 x}{\sqrt{(2 \nu+1)^{2}-4 x^{2}}+2 \nu+1}\right) .
$$

Since $\lim _{x \rightarrow 0^{+}} t(x)=0$, whereas $\lim _{x \rightarrow 0^{+}}\left(-\left(\sqrt{(2 \nu+1)^{2}-4 x^{2}}+2 \nu+1\right) / 2 x\right)=-\infty$, we get

$$
t(x) \geqslant-\frac{2 x}{\sqrt{(2 \nu+1)^{2}-4 x^{2}}+2 \nu+1} \geqslant-\frac{2 x}{2 \nu+1} .
$$

Proof of Theorem 2. By the previous lemma we have

$$
\ln \frac{\mathcal{J}_{\nu}(\nu)}{\mathcal{J}_{\nu}(x)} \geqslant-\int_{x}^{\nu} \frac{2 z}{2 \nu+1} d z=-\frac{\nu^{2}-x^{2}}{2 \nu+1},
$$

hence

$$
\mathcal{J}_{\nu}(t \nu) \leqslant \mathcal{J}_{\nu}(\nu) \exp \left(\frac{\nu^{2}\left(1-t^{2}\right)}{2 \nu+1}\right)
$$

This, together with the inequality $J_{\nu}(\nu)<2^{1 / 3} /\left(3^{2 / 3} \Gamma(2 / 3) \nu^{1 / 3}\right)$ (see [9, equation (10.14.2)]), yields the required result.

Remark 1. The function $\mathcal{J}_{\nu}(x)=x^{-\nu} J_{\nu}(x)$ of Lemma 11 belongs to the so-called PólyaLaguerre class and satisfies the infinite series of inequalities

$$
L_{m}\left(\mathcal{J}_{\nu}\right)=\sum_{j=0}^{2 m}(-1)^{m+j} \frac{\left(\begin{array}{c}
2 m \\
j
\end{array}\right)}{(2 m) !} \mathcal{J}_{\nu}^{(j)} \mathcal{J}_{\nu}^{(2 m-j)} \geqslant 0
$$

where $L_{1}\left(\mathcal{J}_{\nu}\right) \geqslant 0$ is the usual Laguerre inequality $\mathcal{J}_{\nu}^{\prime 2}-\mathcal{J}_{\nu} \mathcal{J}_{\nu}^{\prime \prime} \geqslant 0$ (see, for example, [11, 12]). Using $L_{m}\left(\mathcal{J}_{\nu}\right) \geqslant 0$ for $m>1$ leads to much more precise yet more complicated bounds on $\mathcal{J}_{\nu}^{\prime} / \mathcal{J}_{\nu}$ and consequently on $J_{\nu}$. Alternatively, one can use the inequality $L_{1}\left(\mathcal{J}_{\nu}+\lambda \mathcal{J}_{\nu}^{\prime}\right) \geqslant 0$, $\lambda \in \mathbb{R}$, then optimizing in $\lambda$. It is worth noticing that both methods give an inequality similar to (23) but in the opposite direction. Thus, at least in principal, one can use the known value of $\mathcal{J}_{\nu}(0)$ instead of $\mathcal{J}_{\nu}(\nu)$.

Our main tool for bounding solutions of the second order differential equations will be Sonin's function. In particular, it was used by Szegö to prove that

$$
\left|J_{\nu}(x)\right| \leqslant \sqrt{\frac{2}{\pi x}}, \quad|\nu| \leqslant \frac{1}{2}, x>0 .
$$


Although he did not state this explicitly, his proof of Theorem 7.31.2 from [14] immediately implies

$$
\left|Y_{\nu}(x)\right| \leqslant \sqrt{\frac{2}{\pi x}}, \quad|\nu| \leqslant \frac{1}{2}, x>0 .
$$

His arguments go as follows: let $y$ be a solution of the Bessel differential equation

$$
x^{2} y^{\prime \prime}+x y^{\prime}+\left(x^{2}-\nu^{2}\right) y=0,
$$

the normal form of which is given by

$$
f^{\prime \prime}+\left(1-\frac{\nu^{2}-\frac{1}{4}}{x^{2}}\right) f=0, \quad f=\sqrt{x} y
$$

Then for $|\nu| \leqslant \frac{1}{2}$ and $x>0$ the derivative of Sonin's function is positive,

$$
S^{\prime}(x)=\frac{2 \mu x}{\left(\mu+x^{2}\right)^{2}} f^{\prime 2}(x)>0,
$$

hence $S$ is increasing and inequalities (25) and (26) follow by calculating $S(\infty)$ from known asymptotics of $J_{\nu}$ and $Y_{\nu}$. On the other hand, $S^{\prime}(x)<0$ for $\nu>\frac{1}{2}$ and $x>\sqrt{\mu}$, which does not lead, at least directly, to any explicit inequality.

It turns out that for $\nu>\frac{1}{2}$ it is more natural to deal with the function

$$
\mathcal{H}_{\nu}(x)=\left|x^{2}-\mu\right|^{1 / 4} J_{\nu}(x),
$$

rather than $\sqrt{x} J_{\nu}(x)$. Here we will refine an inequality for the Bessel function obtained in [2]. First we need the following lemma.

Lemma 12. The first positive maximum of $\mathcal{H}_{\nu}(x), \nu \geqslant \frac{5}{3}$, is attained at a point $\xi$ satisfying

$$
\xi>\nu \sqrt{1-(2 \nu)^{-2 / 3}} \text {. }
$$

Proof. Since obviously $0<\xi<\mu$, we can restrict ourselves to the interval $(0, \mu)$ and write down

$$
\mathcal{H}_{\nu}(x)=x^{\nu}\left(\mu-x^{2}\right)^{1 / 4} \mathcal{J}_{\nu}(x),
$$

where as before $\mathcal{J}_{\nu}(x)=x^{-\nu} J_{\nu}(x)$. Then

$$
0=\mathcal{H}_{\nu}^{\prime}(\xi)=\frac{\xi^{\nu-1}}{2\left(\mu-\xi^{2}\right)^{3 / 4}}\left(2\left(\mu-\xi^{2}\right)(\xi t(\xi)+\nu)-\xi^{2}\right) \mathcal{J}_{\nu}(\xi)
$$

where $t(x)=\mathcal{J}_{\nu}^{\prime}(x) / \mathcal{J}_{\nu}(x)$. Hence

$$
t(\xi)=-\frac{(2 \nu+1)\left(2 \nu^{2}-\nu-2 \xi^{2}\right)}{\left(4 \nu^{2}-1-4 \xi^{2}\right) \xi},
$$

and comparing this with (4) we obtain the inequality

$$
\frac{(2 \nu+1)\left(2 \nu^{2}-\nu-2 \xi^{2}\right)}{4 \nu^{2}-1-4 \xi^{2}} \leqslant \frac{2 \nu+1-\sqrt{(2 \nu+1)^{2}-4 \xi^{2}}}{2} .
$$

Simplifying we get that the last inequality holds if

$$
p(\xi)=\left(4 \nu^{2}-1\right)^{2}-\left(4 \nu^{2}-1-\xi^{2}\right)^{2}\left((2 \nu+1)^{2}-4 \xi^{2}\right) \geqslant 0 .
$$


Observe that for $\nu \geqslant \frac{5}{3}$ this polynomial has the only positive zero $\xi_{0}$. Indeed, the discriminant of $p(\xi)$ in $\xi$, up to an irrelevant numerical factor, is

$$
\nu(\nu+1)(2 \nu-1)^{6}(2 \nu+1)^{10}\left(108 \nu^{2}-172 \nu-5\right)^{2} .
$$

Thus the number of real and, as it is an even function of $\xi$, positive zeros does not change for $108 \nu^{2}-172 \nu-5>0$, in particular for $\nu>\frac{5}{3}$. Choosing $\nu=\frac{5}{2}$ we obtain the test equation $p(\xi)=\xi^{6}-21 \xi^{4}+144 \xi^{2}-315=0$, with the only positive zero $\xi \approx 2.14$. Finally,

$$
p(\nu)=\nu\left(4 \nu^{3}-2 \nu-1\right)>0,
$$

and using the substitution $\nu=r^{3} / 2$, we find

$$
p\left(\nu \sqrt{1-(2 \nu)^{-2 / 3}}\right)=p\left(\sqrt{r^{6}-r^{4}} / 2\right)=-r^{3}\left(r^{2}-1\right)^{2}\left(2 r^{4}+4 r^{2}-r+2\right)<0 .
$$

Hence, since $r>1$, we get $\xi>\nu \sqrt{1-(2 \nu)^{-2 / 3}}$.

Proof of Theorem 3. For $x=\sqrt{\mu}$ the result is trivial. Otherwise we shall consider three (in fact, overlapping) cases.

Case 1: $x>\sqrt{\mu}$. The function $\mathcal{H}_{\nu}(x)=\left(x^{2}-\mu\right)^{1 / 4} J_{\nu}(x)$, as is easy to check, satisfies the differential equation

$$
\mathcal{H}_{\nu}^{\prime \prime}(x)-\frac{\mu}{x\left(x^{2}-\mu\right)} \mathcal{H}_{\nu}^{\prime}(x)+\frac{4\left(x^{2}-\mu\right)^{3}+\left(6 x^{2}-\mu\right) \mu}{4 x^{2}\left(x^{2}-\mu\right)^{2}} \mathcal{H}_{\nu}(x)=0 .
$$

Consider the corresponding Sonin's function

$$
S(x)=\mathcal{H}_{\nu}^{2}(x)+\frac{4 x^{2}\left(x^{2}-\mu\right)^{2}}{4\left(x^{2}-\mu\right)^{3}+\left(6 x^{2}-\mu\right) \mu} \mathcal{H}^{\prime 2}(x),
$$

then $\mathcal{H}_{\nu}^{2}(x) \leqslant S(x)$ for $x>\sqrt{\mu}>0$. On excluding $\mathcal{H}_{\nu}^{\prime \prime}(x)$ by $(28)$ one finds

$$
S^{\prime}(x)=\frac{24 \mu x^{3}\left(x^{2}-\mu\right)\left(4 x^{2}+\mu\right)}{\left(4\left(x^{2}-\mu\right)^{3}+\left(6 x^{2}-\mu\right) \mu\right)^{2}} \mathcal{H}^{\prime 2}(x) \geqslant 0,
$$

hence $\left|\mathcal{H}_{\nu}(x)\right|<\sqrt{\lim _{x \rightarrow \infty} S(x)}$. Using $J_{\nu}^{\prime}(x)=\left(J_{\nu-1}(x)-J_{\nu+1}(x)\right) / 2$, and the asymptotic formula

$$
J_{\nu}(x) \sim \sqrt{\frac{2}{\pi x}} \cos \left(x-\omega_{\nu}\right)
$$

after some calculations one finds $\left|\mathcal{H}_{\nu}(x)\right|<\sqrt{2 / \pi}$. Since $\mathcal{H}_{\nu}^{2}(x)=S(x)$ at all local maxima the constant $\sqrt{2 / \pi}$ is sharp.

Case 2 : $0<x \leqslant \sqrt{\mu}, \frac{1}{2} \leqslant \nu \leqslant 4.9$. By $(22)$ and $\nu \geqslant \frac{1}{2}$ we have

$$
\mathcal{H}_{\nu}(x)=\left(\mu-x^{2}\right)^{1 / 4} J_{\nu}(x) \leqslant \frac{\left(\mu-x^{2}\right)^{1 / 4} x^{\nu}}{2^{\nu} \Gamma(\nu+1)} .
$$

The maximum of the right-hand side is attained for $x=\sqrt{\nu^{2}-\nu / 2}$, yielding

$$
\begin{aligned}
\mathcal{H}_{\nu}(x) & \leqslant\left(1-\frac{1}{2 \nu}\right)^{\nu / 2+1 / 4} \frac{(\nu / 2)^{\nu / 2+1 / 4}}{\Gamma(\nu+1)}<e^{-1 / 4} \frac{(\nu / 2)^{\nu / 2+1 / 4}}{\sqrt{2 \pi \nu} \nu^{\nu} e^{-\nu}} \\
& =\frac{(e / 2)^{\nu-1 / 4}}{2 \sqrt{\pi} \nu^{1 / 4}}<\sqrt{2 / \pi}, \quad \frac{1}{2} \leqslant \nu \leqslant 4.9 .
\end{aligned}
$$


Case 3: $0<x<\sqrt{\mu}, \nu \geqslant \frac{19}{7}$. Inequality (4) yields

$$
\mathcal{H}_{\nu}(x)<\frac{2^{1 / 3} x^{\nu}\left(\mu-x^{2}\right)^{1 / 4}}{3^{2 / 3} \Gamma(2 / 3) \nu^{\nu+1 / 3}} \exp \left(\frac{\nu^{2}-x^{2}}{2 \nu+1}\right) .
$$

Let $r=(2 \nu)^{1 / 3}$. By Lemma 12 we can set

$$
x=\nu \sqrt{1-(2 \nu)^{-2 / 3} z}=\frac{r^{2}}{2} \sqrt{r^{2}-z}, \quad r^{-4}<z<1 \leqslant r .
$$

This gives $\mathcal{H}_{\nu}(x)<A f(z)$, where

$$
A=\frac{2^{1 / 6}}{3^{2 / 3} \Gamma(2 / 3)}, \quad f(z)=\left(1-\frac{z}{r^{2}}\right)^{r^{3} / 4}\left(z-\frac{1}{r^{4}}\right)^{1 / 4} \exp \left(\frac{z r^{4}}{4 r^{3}+4}\right)
$$

We find

$$
\frac{f^{\prime}(z)}{f(z)}=\frac{\left(1+r^{3}+r^{6}-2 z r^{4}-z^{2} r^{5}\right) r^{3}}{4\left(1+r^{2}\right)\left(r^{2}-z\right)\left(z r^{4}-1\right)}
$$

The denominator here is obviously positive. The numerator is also positive by

$$
1+r^{3}+r^{6}-2 z r^{4}-z^{2} r^{5}>\left(r^{2}-1\right)(1+r)\left(r^{3}-2 r^{2}+r-1\right)>0,
$$

provided $\nu \geqslant \frac{19}{7}$. Hence $f(x)$ is increasing and

$$
\begin{aligned}
\mathcal{H}_{\nu}(x)<A f(1) & =A\left(1-\frac{1}{r^{2}}\right)^{r^{3} / 4}\left(r^{4}-1\right)^{1 / 4} r^{-1} \exp \left(\frac{r^{4}}{4 r^{3}+4}\right) \\
& <A e^{-r / 4}\left(1-r^{-4}\right)^{1 / 4} \exp \left(\frac{r^{4}}{4 r^{3}+4}\right)<A<\sqrt{2 / \pi}
\end{aligned}
$$

This completes the proof.

Proof of Theorem 7. Set $f(x)=x^{1 / 4} \operatorname{Ai}(-x)$, then

$$
f^{\prime \prime}-\frac{1}{2 x} f^{\prime}+\left(x+\frac{5}{16 x^{2}}\right) f=0
$$

and the corresponding Sonin's function is

$$
S(x)=f^{2}+\frac{16 x^{2}}{16 x^{3}+5} f^{\prime 2}, \quad S^{\prime}(x)=\frac{240 x}{\left(16 x^{3}+5\right)^{2}} f^{\prime 2} \geqslant 0 .
$$

Hence $f^{2}(x) \leqslant S(x) \leqslant S(\infty)$. Using the asymptotics [9, equations (9.7.9), (9.7.10)]

$$
\operatorname{Ai}(-x) \sim \frac{\cos (\zeta-\pi / 4)}{\sqrt{\pi} x^{1 / 4}}, \quad \operatorname{Ai}^{\prime}(-x) \sim \frac{x^{1 / 4} \sin (\zeta-\pi / 4)}{\sqrt{\pi}}, \quad \zeta=\frac{2}{3} x^{3 / 2},
$$

one obtains $S(\infty)=1 / \pi$ and the result follows.

\section{Error term of the standard asymptotics of $J_{\nu}(x)$}

Having at hand an upper bound on $\left|J_{\nu}(x)\right|$ one can estimate the difference

$$
r(x)=\sqrt{\frac{\pi x}{2}} J_{\nu}(x)-\cos \left(x-\omega_{\nu}\right)
$$


in a rather elementary way. Notice that $r(x)$ satisfies the equation

$$
r^{\prime \prime}+r=\sqrt{\frac{\pi}{2 x^{3}}}\left(\nu^{2}-\frac{1}{4}\right) J_{\nu}(x),
$$

with the general solution of the form

$$
r(x)=c_{1} \cos x+c_{2} \sin x+\sqrt{\frac{\pi}{2}}\left(\frac{1}{4}-\nu^{2}\right) \int_{x}^{\infty} \frac{\sin (t-x)}{t^{3 / 2}} J_{\nu}(t) d t .
$$

Now one has only to estimate the integral and to notice that as far as it is $o(1)$, we have $c_{1}=c_{2}=0$ by an obvious limiting argument.

To get better numerical constants the following observation will be useful. Let $f(x) \geqslant 0$ be a decreasing function for $x>0$. Since

$$
\int_{\pi k}^{\pi(k+1)}(|\sin t|-2 / \pi) d t=0, \quad k=0,1, \ldots
$$

one readily obtains

$$
\int_{0}^{\infty} f(t)|\sin t| d t \leqslant \frac{2}{\pi} \int_{0}^{\infty} f(t) d t
$$

assuming that the last integral exists.

Proof of Theorem 4. We shall estimate the function $r(x)$ defined by (29). First notice that asymptotically

$$
J_{\nu}(x)=\sqrt{\frac{2}{\pi x}} \cos \left(x-\omega_{\nu}\right)+O\left(x^{-3 / 2}\right),
$$

that is $\lim _{x \rightarrow \infty} r(x)=0$, hence $c_{1}=c_{2}=0$ in (30), and therefore

$$
|r(x)|=\sqrt{\frac{\pi}{2}} \mu\left|\int_{x}^{\infty} \frac{\sin (t-x)}{t^{3 / 2}} J_{\nu}(t) d t\right|:=\sqrt{\frac{\pi}{2}} \mu \mathcal{I}_{\nu}(x) .
$$

Thus, $c=\sup _{x>0} x \mathcal{I}_{\nu}(x)$ and for $|\nu| \leqslant \frac{1}{2}$ the result immediately follows by (31),

$$
\mathcal{I}_{\nu}(x) \leqslant \sqrt{\frac{2}{\pi}} \int_{x}^{\infty} \frac{|\sin (t-x)|}{t^{2}} d t \leqslant \frac{(2 / \pi)^{3 / 2}}{x} .
$$

For $\nu>\frac{1}{2}$ and $x>\sqrt{\mu}$, applying (5), (31) and the inequality $\arcsin x \leqslant \pi x / 2$, we get

$$
\begin{aligned}
& \mathcal{I}_{\nu}(x) \leqslant \sqrt{\frac{2}{\pi}} \int_{x}^{\infty} \frac{|\sin (t-x)|}{t^{3 / 2}\left(t^{2}-\mu\right)^{1 / 4}} d t \leqslant\left(\frac{2}{\pi}\right)^{3 / 2} \int_{x}^{\infty} \frac{d t}{t^{3 / 2}\left(t^{2}-\mu\right)^{1 / 4}}
\end{aligned}
$$

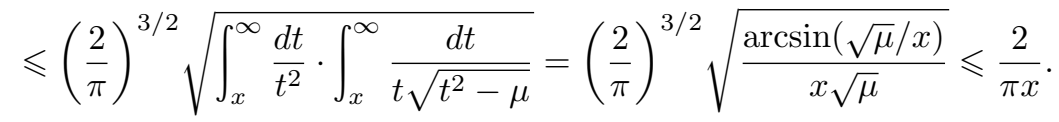

Similarly, for $\nu>\frac{1}{2}$ and $0<x \leqslant \sqrt{\mu}$,

$$
\begin{aligned}
\mathcal{I}_{\nu}(x) & \leqslant \sqrt{\frac{2}{\pi}} \int_{\sqrt{\mu}}^{\infty} \frac{|\sin (t-x)|}{t^{3 / 2}\left(t^{2}-\mu\right)^{1 / 4}} d t+\sqrt{\frac{2}{\pi}} \int_{x}^{\sqrt{\mu}} \frac{|\sin (t-x)|}{t^{3 / 2}\left(\mu-t^{2}\right)^{1 / 4}} d t \\
& \leqslant \frac{2}{\pi \sqrt{\mu}}+\sqrt{\frac{2}{\pi} \int_{x}^{\sqrt{\mu}} \frac{d t}{t^{2}} \cdot \int_{x}^{\sqrt{\mu}} \frac{d t}{t \sqrt{\mu-t^{2}}}}=\frac{2}{\pi \sqrt{\mu}}+\sqrt{\frac{2}{\pi}\left(\frac{1}{x}-\frac{1}{\sqrt{\mu}}\right) \frac{\ln \left(\sqrt{\mu}+\sqrt{\mu-x^{2}}\right) / x}{\sqrt{\mu}} .}
\end{aligned}
$$


This inequality can be rewritten as

$$
x \mathcal{I}_{\nu}(x) \leqslant \frac{2 z}{\pi}+\sqrt{\frac{2}{\pi}(1-z) z \ln \frac{1+\sqrt{1-z^{2}}}{z}} \quad \text { with } z=x / \sqrt{\mu} .
$$

A routine but rather tedious investigation reveals that the last expression does not exceed $\frac{4}{5}$. We omit the details.

Let us show that up to the numerical factor $c$ the error term in (6) is sharp. By (30) and (6) we have for the error term

$$
\begin{aligned}
\mathcal{R}_{\nu}(x) & =x^{3 / 2}\left|J_{\nu}(x)-\sqrt{\frac{2}{\pi x}} \cos \left(x-\omega_{\nu}\right)\right|=\mu x\left|\int_{x}^{\infty} \frac{\sin (t-x)}{t^{3 / 2}} J_{\nu}(t) d t\right| \\
& =\sqrt{\frac{2}{\pi}} \mu x\left|\int_{x}^{\infty} \frac{\sin (t-x) \cos \left(t-\omega_{\nu}\right)}{t^{2}} d t\right|+\theta c \mu x\left|\int_{x}^{\infty} \frac{\sin (t-x)}{t^{3}} d t\right|:=\sqrt{\frac{2}{\pi}} \mu x\left|I_{1}\right|+I_{2} .
\end{aligned}
$$

Here

$$
I_{2}=\theta c \mu x \int_{x}^{\infty} \frac{d t}{t^{3}}=\frac{\theta c \mu}{2 x} .
$$

To bound $I_{1}$ we introduce two auxiliary functions $f$ and $g$ defined by

$$
S i(x)=\frac{\pi}{2}-f(x) \cos x-g(x) \sin x, \quad C i(x)=f(x) \sin x-g(x) \cos x,
$$

with the asymptotics (see $[\mathbf{9}, \S 6.12(\mathrm{ii})])$

$$
f(x)=x^{-1}+O\left(x^{-3}\right), \quad g(x)=x^{-2}+O\left(x^{-4}\right) .
$$

Calculations yield

$$
\begin{aligned}
I_{1} & =S i(2 z) \sin \left(x+\omega_{\nu}\right)+C i(2 z) \cos \left(x+\omega_{\nu}\right)-\left.\frac{\sin t \cos \left(z-\omega_{\nu}\right)}{z}\right|_{t=0} ^{\infty} \\
& =f(2 z) \sin \left(2 t+x-\omega_{\nu}\right)-g(2 z) \cos \left(2 t+x-\omega_{\nu}\right)-\left.\frac{\sin t \cos \left(z-\omega_{\nu}\right)}{z}\right|_{t=0} ^{\infty} \\
& =\frac{\sin \left(\omega_{\nu}-x\right)}{2 x}+O\left(x^{-2}\right), \quad z=x+t
\end{aligned}
$$

Hence

$$
\mathcal{R}_{\nu}(x)=\frac{\mu}{\sqrt{2 \pi}}\left|\sin \left(\omega_{\nu}-x\right)\right|+O\left(x^{-1}\right)
$$

and the result follows.

REMARK 2. Numerical calculations suggest that in fact

$$
\sup _{x \geqslant 0} x^{3 / 2}\left|J_{\nu}(x)-\sqrt{\frac{2}{\pi x}} \cos \left(x-\frac{\pi(2 \nu+1)}{4}\right)\right|=\frac{\mu}{\sqrt{2 \pi}}
$$

for all $\nu \geqslant-\frac{1}{2}$ outside the strip $\frac{3}{2}<\nu \lesssim 4.4767$. The maximal value of the function in that strip is approximately equal to $1.06424 / \sqrt{2 \pi}$ and is attained at the point $(\nu, x) \approx$ (2.687 29, 2.982 19). 


\section{Sharper asymptotics}

The classical asymptotics given by (6) do not make much sense for $x=O(\mu)$ when the main term and the error are of the same order. Here, using formula (21), we derive a different asymptotic expression with much smaller error term. It also leads to very sharp approximation of the Airy function $\mathrm{Ai}(-x)$ and its zeros. We will need the following lemma given in [7].

Lemma 13. Let $f(x)$ satisfy the differential equation

$$
f^{\prime \prime}(x)+b^{2}(x) f(x)=0,
$$

where $b(x)>0$ and $b^{\prime \prime}(x)$ exists on an interval $\mathcal{I}$. Let $g(x)=\sqrt{b(x)} f(x)$, then for $x \in \mathcal{I}$, provided the integral exists,

$$
g(x)=c_{1} \cos \mathcal{B}(x)+c_{2} \sin \mathcal{B}(x)+\theta \int_{a}^{x}\left|\frac{3 b^{\prime 2}(t)-2 b(t) b^{\prime \prime}(t)}{4 b^{3}(t)} g(t)\right| d t
$$

where $\mathcal{B}(x)=\int^{x} b(t) d t$ and $a \in \mathcal{I}$ is arbitrary.

Proof. Observe that $g(x)$ satisfies the equation

$$
g^{\prime \prime}-\frac{b^{\prime}}{b} g^{\prime}+g b^{2}(1+\epsilon)=0, \quad \epsilon=\epsilon(x)=\frac{3 b^{2}-2 b b^{\prime \prime}}{4 b^{4}} .
$$

The solution of the corresponding homogeneous equation

$$
g_{0}^{\prime \prime}-\frac{b^{\prime}}{b} g_{0}^{\prime}+b^{2} g_{0}=0
$$

is $g_{0}=c_{1} \sin \mathcal{B}(x)+c_{2} \cos \mathcal{B}(x)$. Solving (33) formally as an inhomogeneous equation with the right-hand side $-\epsilon(x) g(x)$ we get

$$
\begin{aligned}
g(x) & =g_{0}(x)-\int_{a}^{x} \epsilon(t) b(t) g(t) \sin (\mathcal{B}(x)-\mathcal{B}(t)) d t \\
& =g_{0}(x)+\theta \int_{a}^{x}|\epsilon(t) b(t) g(t) \sin (\mathcal{B}(x)-\mathcal{B}(t))| d t \\
& =g_{0}(x)+\theta \int_{a}^{x}\left|\frac{3 b^{\prime 2}(t)-2 b(t) b^{\prime \prime}(t)}{4 b^{3}(t)} g(t)\right| d t
\end{aligned}
$$

The normal form of differential equation (2) is

$$
f^{\prime \prime}+\left(1-\frac{\nu^{2}-1 / 4}{x^{2}}\right) f=0, \quad f=\sqrt{x} J_{\nu}(x) .
$$

Thus, for $x>\sqrt{\max \left\{0, \nu^{2}-\frac{1}{4}\right\}}$ we have

$$
\begin{aligned}
& b(x)=\frac{\sqrt{x^{2}-\nu^{2}+1 / 4}}{x}, \\
& \mathcal{B}(x)= \begin{cases}\sqrt{x^{2}+\mu}+\sqrt{\mu} \ln \frac{x}{\sqrt{\mu}+\sqrt{\mu+x^{2}}}, & |\nu| \leqslant 1 / 2, \\
\sqrt{x^{2}-\mu}+\sqrt{\mu} \arcsin \frac{\sqrt{\mu}}{x}, & \nu \geqslant 1 / 2,\end{cases}
\end{aligned}
$$

and $g(x)=\left(x^{2}-\nu^{2}+\frac{1}{4}\right)^{1 / 4} J_{\nu}(x)$. 
Proof of Theorem 5. Since $\left|J_{\nu}(x)\right| \leqslant \sqrt{2 / \pi x}$ for $|\nu| \leqslant \frac{1}{2}$, by (32) we have

$$
g(x)=g_{0}(x)+\theta \frac{\mu}{\sqrt{8 \pi}} \int_{x}^{\infty} \frac{6 z^{2}+\mu}{z^{3 / 2}\left(z^{2}+\mu\right)^{9 / 4}} d z=g_{0}(x)+\theta \frac{\mu}{\sqrt{2 \pi x}\left(x^{2}+\mu\right)^{5 / 4}} .
$$

Comparing this with the standard asymptotics

$$
f(x)=\sqrt{x} J_{\nu}(x)=\sqrt{\frac{2}{\pi}} \cos \left(x-\omega_{\nu}\right)+O\left(x^{-3 / 2}\right),
$$

for large $x$ one finds

yielding (8).

$$
c_{1}=\sqrt{\frac{2}{\pi}} \sin \omega_{\nu}, \quad c_{2}=\sqrt{\frac{2}{\pi}} \cos \omega_{\nu}
$$

Similarly, for $\nu \geqslant \frac{1}{2}$ and $x \geqslant \mu$, using (5) instead of (25), we obtain

$$
g(x)=g_{0}(x)+\frac{\theta \mu}{\sqrt{8 \pi}} \int_{x}^{\infty} \frac{6 z^{2}-\mu}{z\left(z^{2}-\mu\right)^{5 / 2}} d z=g_{0}+\frac{\theta}{\sqrt{8 \pi}}\left|\frac{3 x^{2}+2 \mu}{3\left(x^{2}-\mu\right)^{3 / 2}}-\frac{\arcsin (\sqrt{\mu} / x)}{\sqrt{\mu}}\right| .
$$

It is easy to check that the expression inside the absolute value bars is positive and decreasing in $x$. Therefore, by $\arcsin (\sqrt{\mu} / x) \geqslant \sqrt{\mu} / x, x>0$, we get

$$
\begin{aligned}
& \frac{3 x^{2}+2 \mu}{3\left(x^{2}-\mu\right)^{3 / 2}}-\frac{\arcsin (\sqrt{\mu} / x)}{\sqrt{\mu}} \leqslant \frac{3 x^{2}+2 \mu}{3\left(x^{2}-\mu\right)^{3 / 2}}-\frac{1}{x} \\
& \quad=\frac{13 \mu}{6\left(x^{2}-\mu\right)^{3 / 2}}-\frac{\left(x+2 \sqrt{x^{2}-\mu}\right)\left(\sqrt{x^{2}-\mu}-x\right)^{2}}{2 x\left(x^{2}-\mu\right)^{3 / 2}}<\frac{13 \mu}{6\left(x^{2}-\mu\right)^{3 / 2}},
\end{aligned}
$$

and

$$
g(x)=g_{0}(x)+\theta \frac{13 \mu}{12 \sqrt{2 \pi}\left(x^{2}-\mu\right)^{3 / 2}} .
$$

Comparing this with the asymptotics for large $x$ one finds $c_{1}=\sqrt{2 / \pi} \sin \omega_{\nu}, c_{2}=\sqrt{2 / \pi} \cos \omega_{\nu}$, and (9) follows.

The corresponding results for the Airy function are now almost straightforward. In particular, Corollary 2 follows directly from (8).

Proof of Theorem 8. Let $f(x)=\operatorname{Ai}(-x)$, then $f^{\prime \prime}+x f=0$, that is $b(x)=\sqrt{x}$, then $(32)$ together with (14) yield

$$
\begin{aligned}
g(x) & =x^{1 / 4} f(x)=c_{1} \sin \zeta+c_{2} \cos \zeta+\int_{x}^{\infty} \frac{5}{12 \pi x^{3 / 2}} d x \\
& =c_{1} \sin \zeta+c_{2} \cos \zeta+\theta \frac{5}{24 \pi x^{3 / 2}}, \quad \zeta=\frac{2 x^{3 / 2}}{3}
\end{aligned}
$$

and the result follows by comparing this with the asymptotics

$$
\operatorname{Ai}(-x) \sim \frac{1}{\sqrt{\pi} x^{1 / 4}} \cos (\zeta-\pi / 4)
$$

Proof of Theorems 6 and 9. It is easy to verify the following Taylor expansions:

$$
\begin{gathered}
\sqrt{x^{2}+\mu}+\sqrt{\mu} \ln \frac{x}{\sqrt{\mu}+\sqrt{\mu+x^{2}}}=x-\frac{\mu}{2 x}+\theta^{2} \frac{\mu^{2}}{24 x^{3}}, \quad x>0, \\
\frac{\sqrt{16 x^{3}+5}}{6}-\frac{\sqrt{5}}{6} \ln \frac{\sqrt{16 x^{3}+5}+\sqrt{5}}{4 x^{3 / 2}}=\frac{2}{3} x^{3 / 2}-\frac{5}{48} x^{-3 / 2}+\theta^{2} \frac{25}{9216} x^{-9 / 2} .
\end{gathered}
$$

Now (12) and (17) follow by applying $|\cos (x+\epsilon)-\cos x| \leqslant \epsilon, \epsilon \geqslant 0$. 


\section{Approximation of zeros}

In this section we deduce the approximations of Corollary 1 and Theorem 10 from (12) and (17) respectively. Both proofs are based on the following simple observation: the inequality $|\sin x| \leqslant \epsilon$ implies $x=\pi s+\theta \pi \epsilon / 2, s \in \mathbb{Z}$.

Proof of Corollary 1. Let

$$
x_{0}=\frac{r+\sqrt{r^{2}+32 \mu}}{8}, \quad x_{0}^{ \pm}=\frac{r+\sqrt{r^{2}+32 \mu \pm\left(800 \pi \mu / 3 r^{2}\right)}}{8} .
$$

We will prove a slightly stronger result, namely that $x_{0}^{-}<j_{\nu s}<x_{0}^{+}$. Then (13) will follow in view of the inequality

$$
\left|r / 4+2 \mu / r-x_{0}^{ \pm}\right|<18 \pi \mu r^{-3} .
$$

First, notice that $\pi / 2 \leqslant j_{\nu 1} \leqslant \pi$ for $|\nu| \leqslant \frac{1}{2}$, thus we will assume $x>1$. By (12) the equation $J_{\nu}(x)=0$ is equivalent to

$$
\sin \left(x-\frac{\mu}{2 x}-\omega_{\nu}+\frac{\pi}{2}\right)=\theta \frac{25 \mu}{48 x^{3}}
$$

Hence, for $x=j_{\nu s}$ we get

$$
x-\frac{\mu}{2 x}-\omega_{\nu}+\frac{\pi}{2}=\pi s+\theta \frac{25 \pi \mu}{96 x^{4}}
$$

giving the equation

$$
V(x)=96 x^{4}-24 r x^{3}-48 \mu x^{2}-25 \theta \pi \mu=0, \quad r=(4 s+2 \nu-1) \pi .
$$

By Descartes' rule of signs this equation has only one positive zero for $\theta>0$ and a maximum of two for $\theta<0$. If $\theta=0$ then $x_{0}$ is the only positive root of (35).

Suppose next that $\theta>0$. One easily checks $V\left(x_{0}\right)<0$ and $V\left(x_{0}^{+}\right)>0$, hence $x_{0}<x<x_{0}^{+}$.

Now let $\theta<0$. We find $V(0)>0$ and $V\left(\frac{1}{2}\right)<0$. Hence the equation $V(x)=0$ has just one root for $x \geqslant \frac{1}{2}$. Since $V\left(x_{0}\right) \geqslant 0$, whereas

$$
\begin{aligned}
V\left(x_{0}^{-}\right) & =\frac{25 \pi \mu}{6 r^{4}}\left(400 \pi \mu-48 \mu r^{2}-3(1+2 \theta) r^{4}-3 r^{3} \sqrt{r^{2}+32 \mu-\frac{800 \pi \mu}{3 r^{2}}}\right) \\
& \leqslant \frac{25 \pi \mu}{2 r}\left(r-\sqrt{r^{2}+32 \mu-\frac{800 \pi \mu}{3 r^{2}}}\right) \leqslant 0,
\end{aligned}
$$

we conclude that in all the cases $x_{0}^{-}<x<x_{0}^{+}$. This completes the proof.

Proof of Theorem 10. By (17) $\operatorname{Ai}(-x)=0$ means

$$
\sin \left(\frac{2}{3} x^{3 / 2}-\frac{5}{48} x^{-3 / 2}+\frac{\pi}{4}\right)=\theta \frac{5 \pi}{36} x^{-9 / 2}
$$

hence for $x=a_{s}$ we obtain

$$
\frac{2}{3} x^{3 / 2}-\frac{5}{48} x^{-3 / 2}=\pi s-\frac{\pi}{4}+\theta \frac{5 \pi}{36} x^{-9 / 2}, \quad s=1,2, \ldots
$$

First we need a lower bound on $x$. Since $a_{1}=2.33 \ldots$, we may assume $x>2$. Then

$$
\frac{2}{3} x^{3 / 2}=\pi s-\frac{\pi}{4}+\frac{5}{48} x^{-3 / 2}+\theta \frac{5 \pi}{36} x^{-9 / 2}>\pi s-\frac{\pi}{4},
$$


so $x>m^{2 / 3} / 4, m=(12 s-3) \pi$. Substituting this into the term with $\theta$ in (36) we get the equation

$$
\frac{2}{3} x^{3 / 2}-\frac{5}{48} x^{-3 / 2}=m / 12+\delta, \quad \delta=\theta \frac{640 \pi}{9 m^{3}},
$$

with the only positive root

$$
x=16^{-2 / 3}\left(m+12 \delta+\sqrt{(m+12 \delta)^{2}+40}\right)^{2 / 3} .
$$

After some calculations one gets

$$
x=16^{-2 / 3}\left(m+\sqrt{m^{2}+40}\right)^{2 / 3}\left(1+\theta \frac{16 \delta}{m+\sqrt{m^{2}+40}}\right) .
$$

Finally, using the inequality

$$
0<\frac{1}{4}\left(m^{2}+20\right)^{1 / 3}-16^{-2 / 3}\left(m+\sqrt{m^{2}+40}\right)^{2 / 3}<\frac{25}{3 m^{3}\left(m^{2}+40\right)^{1 / 6}},
$$

we get

$$
x=\frac{1}{4}\left(m^{2}+20\right)^{1 / 3}+\theta \frac{457}{m^{3}\left(m^{2}+40\right)^{1 / 6}} .
$$

This completes the proof.

Finally, let us notice that the formulas (37) and (18) can be strengthened to

$$
a_{s}<16^{-2 / 3}\left(m+\sqrt{m^{2}+40}\right)^{2 / 3}<\frac{1}{4}\left(m^{2}+20\right)^{1 / 3} .
$$

This follows from rather involved estimates given in [13] of the error term of Miller's asymptotic expansion for $a_{s}$,

$$
a_{s} \sim m^{2 / 3}\left(\frac{1}{4}+\frac{5}{3 m^{2}}-\frac{1280}{9 m^{4}}+\frac{4936000}{81 m^{6}}-\cdots\right) .
$$

In particular, in $[\mathbf{1 3}]$ it is shown that

$$
a_{s}<m^{2 / 3}\left(\frac{1}{4}+\frac{5}{3 m^{2}}-\frac{1280}{9 m^{4}}+\frac{4936000}{81 m^{6}}\right),
$$

and by straightforward calculations we conclude that the last expression does not exceed $16^{-2 / 3}\left(m+\sqrt{m^{2}+40}\right)^{2 / 3}$.

\section{References}

1. S. Breen, 'Uniform upper and lower bounds on the zeros of Bessel functions of the first kind', J. Math. Anal. Appl. 196 (1995) 1-17.

2. I. M. Fabbri, A. Lucianetti and I. Krasikov, 'On a Sturm Liouville periodic boundary values problem', Integral Transforms Spec. Funct. 20 (2009) 353-364.

3. L. Gatteschi and C. Giordano, 'Error bounds for McMahon's asymptotic approximations of the zeros of the Bessel functions', Integral Transforms Spec. Funct. 10 (2000) 41-56.

4. I. Krasikov, 'Uniform bound for Bessel function', J. Appl. Anal. 12 (2006) 83-92.

5. I. Krasikov, 'An upper bound on Jacobi Polynomials', J. Approx. Theory 149 (2007) 116-130.

6. I. Krasikov, 'On Erdélyi-Magnus-Nevai conjecture for Jacobi polynomials', Constr. Approx. 28 (2008) $113-125$.

7. I. KRASIKOV and A. ZARKh, 'Equioscillatory properties of the Laguerre polynomials', J. Approx. Theory 162 (2010) 2021-2047.

8. A. Ya. Olenko, 'Upper bound on $\sqrt{x} J_{\nu}(x)$ and its applications', Integral Transforms Spec. Funct. 17 (2006) 455-467. 
9. F. W. J. Olver, D. W. Lozier, R. F. Boisvert and C. W. Clark (eds), NIST Handbook of Mathematical Functions (Cambridge University Press, 2010).

10. R. B. PARIS, 'An inequality for the Bessel function $J_{\nu}(\nu x)^{\prime}$, SIAM J. Math. Anal. 15 (1984) 203-205.

11. M. L. PAtrick, 'Some inequalities concerning Jacobi Polynomials', SIAM J. Math. Anal. 2 (1971) 213-220.

12. M. L. PAtrick, 'Extensions of inequalities of the Laguerre and Turán type', Pacific J. Math. 44 (1973) 675-682.

13. G. Pittaluga and L. Sacripante, 'Inequalities for the zeros of the Airy functions', SIAM J. Math. Anal. 22 (1991) 260-267.

14. G. Szegö, Orthogonal polynomials, Colloqium Publications 23 (American Mathematical Society, Providence, RI, 1975).

15. G. N. Watson, A Treatise on the theory of Bessel function (Cambridge University Press, London, 1944).

\author{
Ilia Krasikov \\ Department of Mathematical Sciences \\ Brunel University \\ Uxbridge UB8 $3 \mathrm{PH}$ \\ United Kingdom \\ mastiik@brunel.ac.uk
}

